Estudios Geológicos, 65(2)

julio-diciembre 2009, 133-146

ISSN: 0367-0449

doi:10.3989/egeol.39972.075

\title{
Estructura de los mármoles alpujárrides situados entre Calahonda y Castell de Ferro (Cordillera Bética, provincia de Granada)
}

\author{
Structure of the Alpujarride marbles situated between Calahonda \\ and Castell de Ferro (Betic Cordillera, province of Granada)
}

\author{
C. Sanz de Galdeano ${ }^{1}$
}

\begin{abstract}
RESUMEN
Los mármoles de Calahonda-Castell de Ferro presentan dos sistemas de pliegues: uno de dirección $\mathrm{N} 70^{\circ} \mathrm{E}$ a E-O y otro posterior N-S. Ambos se han formado en condiciones en las que los mármoles tenían una neta plasticidad. La dirección de los primeros pliegues es común en la Zona Interna Bética, ligada a etapas de compresión y emplazamientos de mantos. Los segundos pliegues podrían estar relacionados con el desplazamiento de dicha zona hacia el oeste. Buena parte de las fallas y diaclasas existentes son posteriores e ilustran sobre otras etapas de transcurrencia, exhumación, compresión y extensión. La transcurrencia es la continuación de la traslación hacia el oeste y las siguientes etapas se produjeron durante el Mioceno medio y sobre todo el superior.
\end{abstract}

Palabras clave: Cordillera Bética, Complejo Alpujárride, mármoles, pliegues, fallas.

\begin{abstract}
The marbles of the Calahonda-Castell de Ferro area present two systems of folds: the first one has a $\mathrm{N} 70^{\circ} \mathrm{E}$ to $\mathrm{E}-\mathrm{W}$ direction and the second, according to the observed superposed structures, is N-S. Both systems were deformed under plastic conditions. The strike of the first system is common in the Betic Internal Zone, linked to compression and nappe structuring. The second one, less represented, could be related with the westward drifting of the cited zone. A great number of faults and joints formed in later stages of deformation. The first ones correspond to the continuation of the westward translation of the Internal Zone. The rest of the deformations occurred during the middle Miocene and, particularly, from the late Miocene onwards.
\end{abstract}

Key words: Betic Cordillera, Alpujarride Complex, marbles, folds, faults.

\section{Introducción}

Los mármoles triásicos del Complejo Alpujárride presentan en muchas zonas un aspecto masivo que impide estudiar su estructura interna. Éste ha sido aparentemente el caso de los mármoles situados entre las localidades costeras granadinas de Calahonda y Castell de Ferro. Sin embargo, cuando se observan con detenimiento, en algunos sectores existen diferentes términos litológicos que permiten deducir la estructura y, además, se pueden observar estructuras menores, particularmente en cortes de carretera.
El estudio de los principales rasgos de la estructura de los mármoles de este sector permite además su comparación con la conocida en otros sectores del Complejo Alpujárride, lo que facilita sacar conclusiones sobre las etapas de deformación, sus direcciones y hacer conjeturas sobre la edad de las mismas. Éstos son los objetivos del presente artículo.

El Alpujárride es uno de los complejos tectónicos de la Zona Interna Bética, los cuales están apilados tectónicamente y de abajo arriba son: el Nevado-Filábride, el Alpujárride, el Maláguide y la Dorsal, que generalmente se asocia con el Malágui-

\footnotetext{
1 Instituto Andaluz de Ciencias de la Tierra (CSIC-Univ. Granada). Facultad de Ciencias. Universidad de Granada, 18071. Granada, España.Email: csanz@ugr.es
} 


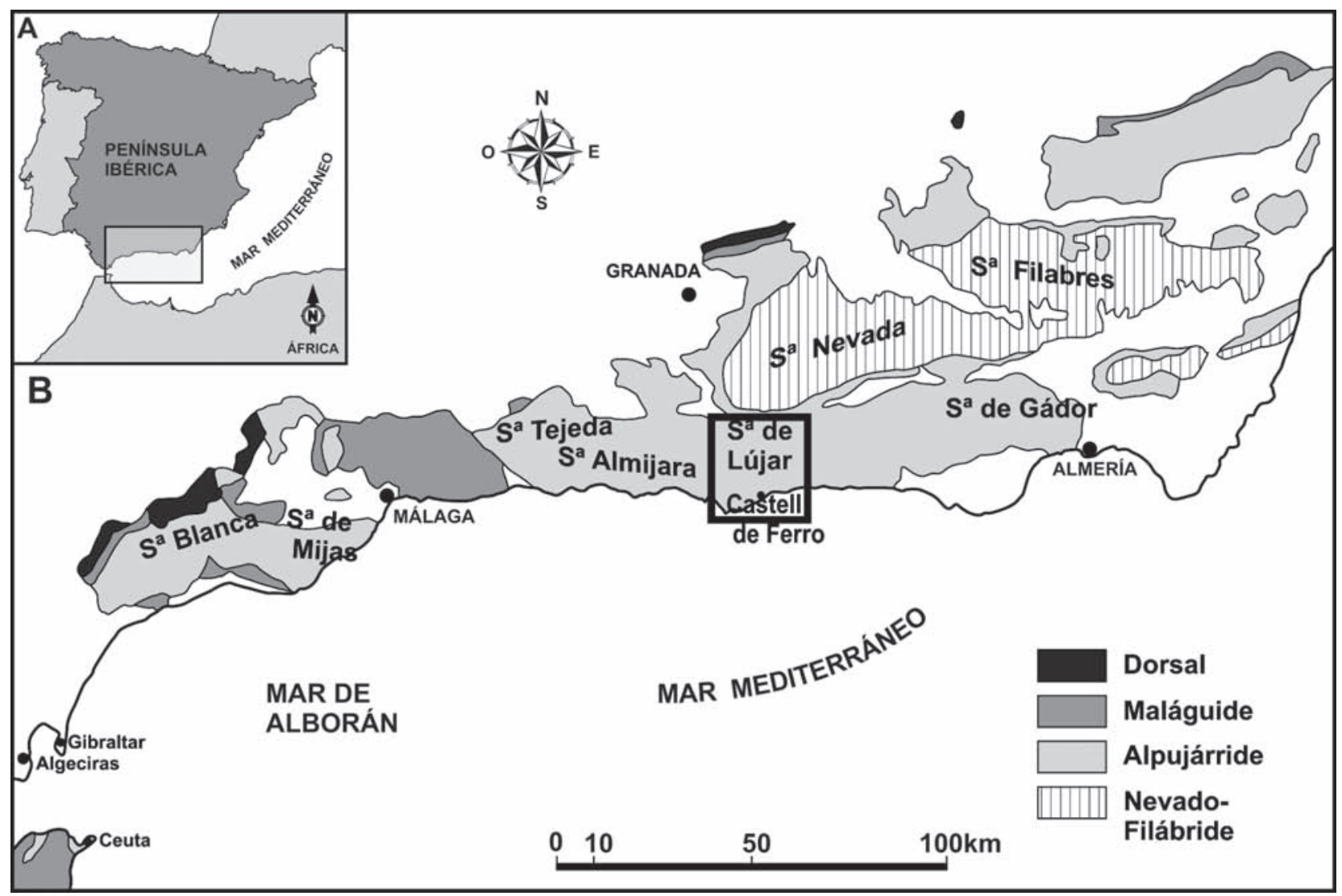

Fig. 1.-A) Situación general del área estudiada en la Cordillera Bética dentro de la península lbérica. Se marca la posición de B. B) Esquema muy simplificado de la distribución de los complejos tectónicos de la Zona Interna Bética. Se marca la posición de la figura 2.

de (fig. 1). De ellos, los dos primeros presentan metamorfismo alpino, que en el caso del área estudiada es el responsable de la marmorización de los sedimentos carbonatados triásicos del Alpujárride.

Estos mármoles de Calahonda (figs. 2 y 3) han sido estudiados de forma muy general tan sólo por Aldaya (1969 y 1981) quien los atribuyó a la unidad de Murtas, una unidad situada en posición intermedia dentro del Complejo Alpujárride (Aldaya \& GarcíaDueñas, 1976; Aldaya et al., 1979; Sanz de Galdeano, 1997). En esos estudios los mármoles de Calahonda siempre han sido considerados de manera indiferenciada, situados encima de las filitas infrayacentes. No parecen existir otros estudios que conciernan directamente a estos mármoles, lo que significa que no hay descripciones previas de su estructura.

\section{Secuencia litológica de la unidad de Murtas}

Esta unidad (equivalente a la de Almijara de Sanz de Galdeano, 1997 y Sanz de Galdeano \&
López-Garrido, 2003) (fig. 2), a la que pertenecen los mármoles estudiados, presenta en su base esquistos pardo-oscuros, con granate y biotita y cuarcitas, y se atribuyen al Paleozoico. No están representados en el sector estudiado sino que aparecen algo más lejos.

Encima existen filitas y cuarcitas. Las filitas generalmente son de color gris azulado pero no siempre es así, de manera que al norte de Castell de Ferro hasta Gualchos y hasta el Pico del Águila (fig. 3) en algunos puntos presentan tonos oscuros que en una primera observación pueden ser confundidas con los esquistos. Quizás sea debido a que, durante su depósito como arcillas y arenas, hubo una cierta concentración de materia orgánica por corresponder a un medio continental lacustre o más bien salobre tipo albufera, aún fuera de modo efímero. Localmente, aunque no se han observado en el área estudiada, hay yesos, lo que indica un medio propicio a la formación de evaporitas. Las cuarcitas se intercalan entre las filitas, si bien muestran una mayor concentración hacia el techo. Su color generalmente es 


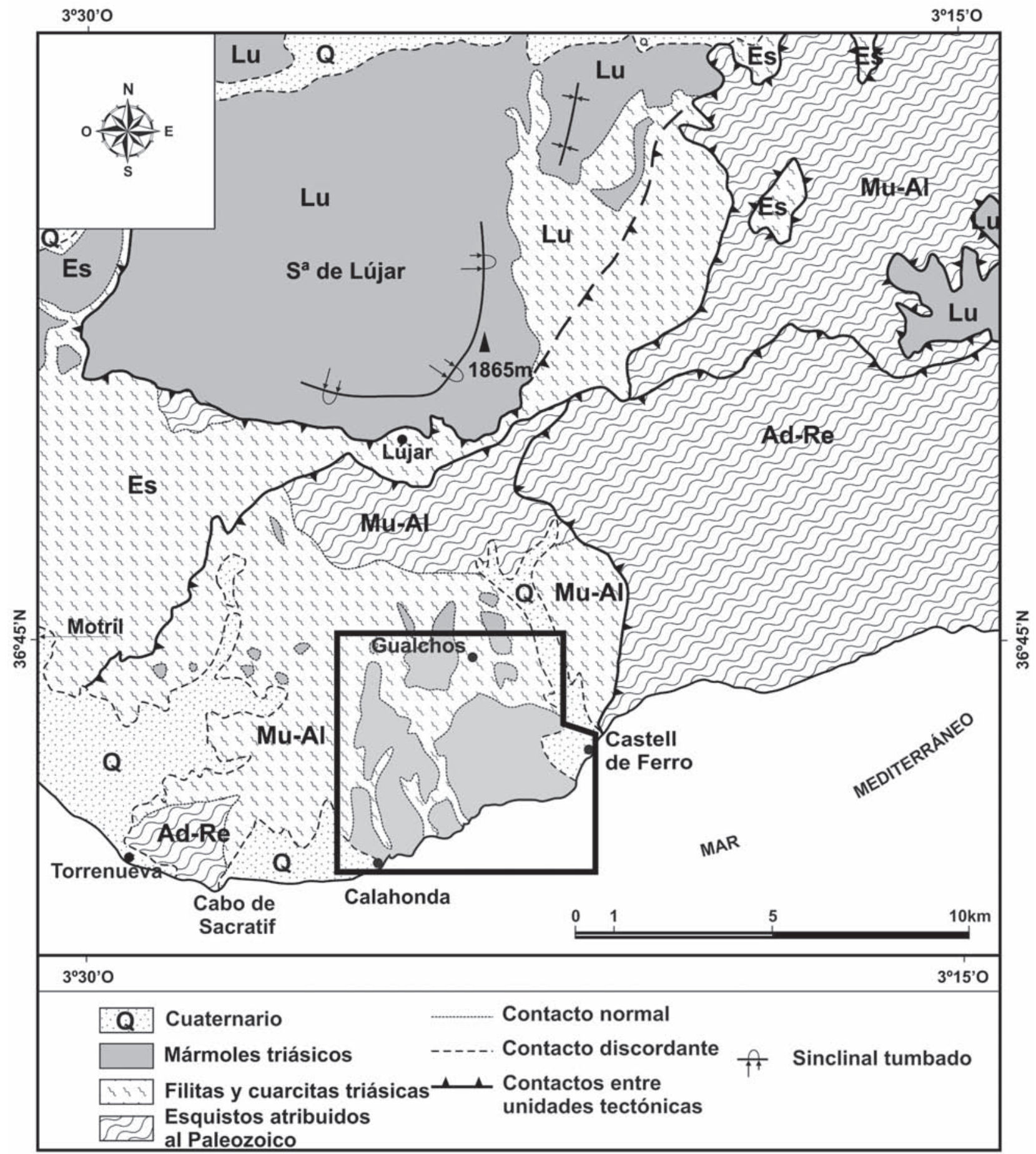

Fig. 2.- Rasgos generales de la distribución de unidades en la región circundante al área estudiada, la cual se señala en el recuadro. Unidades inferiores: Lu: unidad de Lújar. Es: unidad de Escalate. Unidad media: Mu-Al: unidad de Murtas o Almijara. Unidad superior: Ad-Re: unidad Adra o Reales (o Guájares). Se han utilizado datos de Aldaya y García-Dueñas (1976), Aldaya et al. (1979) y Sanz de Galdeano (1997). 


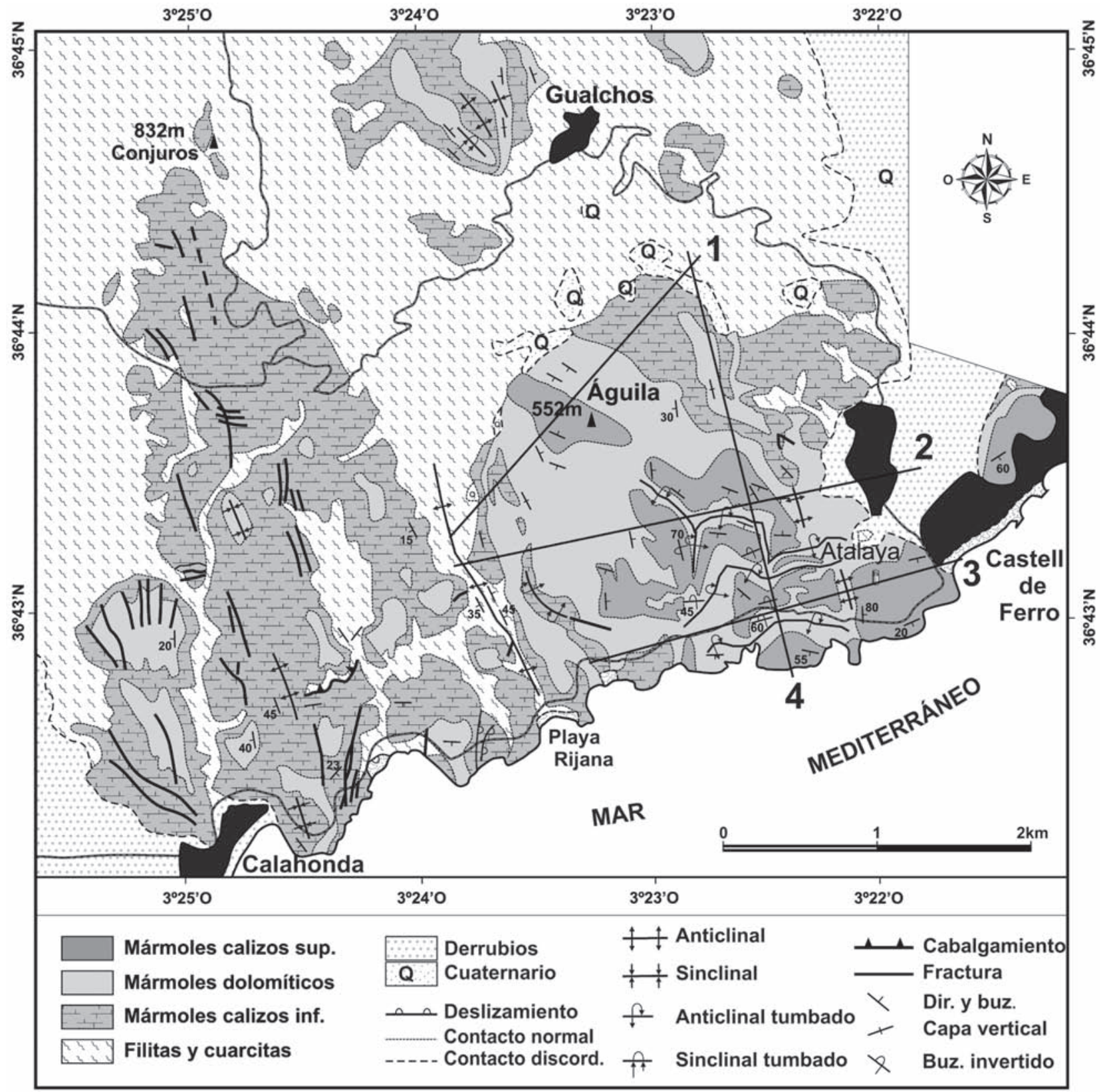

Fig. 3.-Cartografía de los mármoles del sector Calahonda-Castell de Ferro. Su posición se señala en la figura 2.

blanco a amarillento. A techo, en el tránsito a los mármoles existen niveles amarillentos de calcoesquistos, con espesores variables, generalmente del orden de unos $5 \mathrm{~m}$, que en algunos puntos pueden superar los $10 \mathrm{~m}$. Dentro de las filitas existen localmente rocas ígneas de tipo ofita o dolerita.

La edad de las filitas y cuarcitas se atribuye en el Complejo Alpujárride al Trías inferior (Delgado et al., 1981).
Los mármoles se sitúan sobre los calcoesquistos citados y en ellos se distinguen tres tramos litoestratigráficos. El inferior corresponde a mármoles calizos, el medio a dolomíticos y el superior de nuevo está formado por mármoles calizos.

Los mármoles calizos inferiores comienzan alternando con los calcoesquistos y corresponden a antiguas calizas estratificadas en niveles delgados, del orden de 10 a $20 \mathrm{~cm}$, pero que hacia techo van 

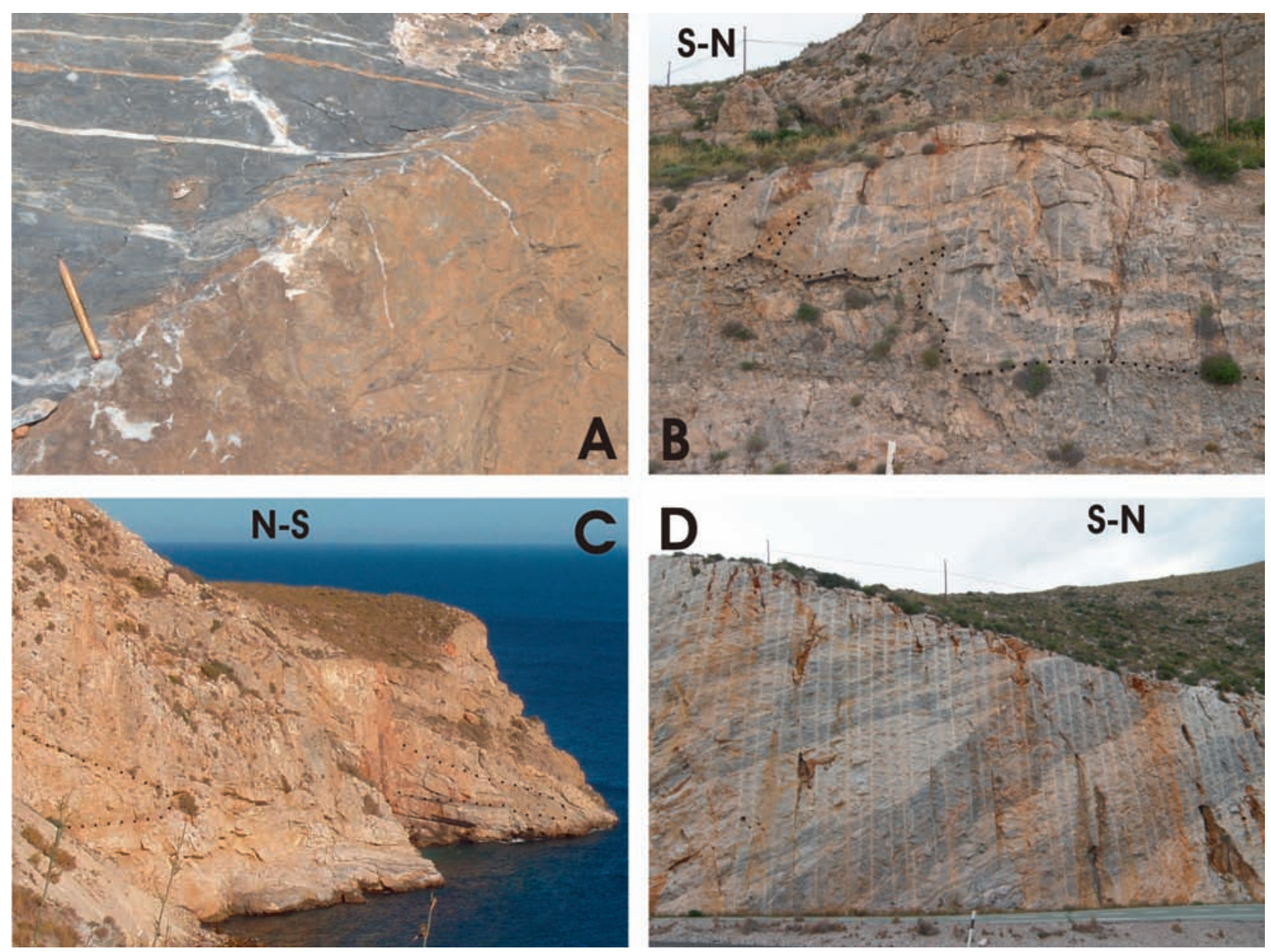

Fig. 4.-A) Detalle de dolomitización del techo de los mármoles inferiores. Afecta a estructuras metamórficas previamente formadas. B) Pliegues al E de Calahonda. Ahí el talud de la carretera está protegido por una red metálica y por eso no es muy nítida la foto. El $\mathrm{N}$ está a la derecha. C) Se marcan dos pliegues sinclinales apretados situados al E de Calahonda. El N está a la izquierda. D) Pliegues en mármoles superiores situados próximos a Castell de Ferro. El N está a la derecha. Todos estos pliegues son vergentes al NNO.

pasando a otros más gruesos, del orden del metro, aunque también hay recurrencias más finas (en realidad estos actuales niveles más que una verdadera estratificación corresponden a la foliación metamórfica, pues su estructura interna está muy deformada como se muestra más adelante. Pero a la vez es cierto que la estratificación original ha controlado en buena medida la disposición y características de la foliación en el conjunto de los mármoles). El límite superior de estos mármoles no suele ser muy definido, pues sus materiales a techo se encuentran parcialmente dolomitizados, de manera que no corresponde a un nivel determinado preciso. Hay localmente «bolsas» dolomíticas intercaladas, y a escala de afloramiento la dolomitización se muestra irregular. Al menos parte de esta dolomitización se ha alcanzado después de la estructuración de los mármoles, pues pequeños pliegues y diaclasas rellenas de calcita se encuentran en algunos puntos incluidos en materiales dolomitizados y lateralmente pasan a otros que siguen siendo calizos (fig. 4A). Dada esta irregularidad que existe en el detalle, la cartografía de la figura 3, particularmente en su sector occidental, es tan sólo una aproximación que, sin embargo, no oscurece los rasgos generales. El espesor promedio calculado de estos mármoles es del orden de 200 a $250 \mathrm{~m}$. Sus tonos son generalmente claros, aunque localmente no sea así, ya que pueden presentar tonos oscuros por alteración, con lo que la separación con el tramo superior dolomítico es también en algunos casos problemática.

Los mármoles dolomíticos están dispuestos en bancos gruesos, del orden del metro (aunque también hay bancos más finos) en los que difícilmente 
se ven estructuras originales, al menos en superficies ampliamente expuestas a la alteración donde casi siempre tienen tonos oscuros; en cortes frescos son de tonos azulados oscuros. Sin embargo, hacia techo se ha observado en algunos cortes niveles poco deformados con restos de algas y dolomías de tipo «franciscano», es decir, alternancia de niveles centimétricos de colores blancos y negros. En estos casos la actual foliación prácticamente coincide con los estratos originales. El espesor estimado de estos mármoles es del orden de 150-200 m.

Sobre los mármoles dolomíticos se sitúan los mármoles calizos superiores. Sus características son similares a los inferiores de manera que si no se lleva un control de la estructura se pueden confundir. Se inician con niveles finos, del orden de 5-10 a $20 \mathrm{~cm}$ que hacia techo se van haciendo más gruesos, de hasta $1 \mathrm{~m}$ de espesor. Cerca de la base presentan en algunos puntos mineralizaciones de hierro que tienen formas que van desde estratoides a cuerpos más o menos masivos que generalmente no tienen longitudes superiores a 30-40 m. El espesor máximo estimado de los mármoles superiores (no existen más tramos a techo) es de unos $150 \mathrm{~m}$.

Mármoles alpujárrides equivalentes a estos tres tramos han sido datados como Trías superior (Delgado et al., 1981; Sanz de Galdeano \& LópezGarrido, 1999).

\section{Estructura de los mármoles}

La estructura se describe con la ayuda de las figuras 3 a 8. Sin duda, el sector más interesante es el que conserva los tres tramos de mármoles descritos, pues es donde se pueden hacer las mejores observaciones, al menos en lo referido a pliegues que son el rasgo más interesante. Ese sector es el que corresponde al cuadrante SE.

Las dimensiones de los pliegues son muy variables. Algunos superan varios kilómetros de longitud en sus ejes y flancos y otros por el contrario son de tamaño centimétrico e incluso menores. Para la descripción de los rasgos principales de la estructura se comienza por los de mayor tamaño.

\section{Los pliegues mayores a decamétricos}

En las figuras 3 y 5 se observa que hay un neto desarrollo de pliegues de dirección NNO-SSE, localmente algo más girado al oeste, tal como es el caso del sinclinal del pico del Águila que es NOSE. En las charnelas anticlinales de estos pliegues afloran niveles de filitas que hacen perder continuidad a los mármoles.

El conjunto de estos pliegues casi N-S se observa en los cortes 1 a 3 de la figura 5 y en ellos se ve que tienen una cierta vergencia hacia el oeste.

Existen también pliegues de dirección aproximada E-O que se conservan sobre todo en el sector SE, de la Atalaya y proximidades. La estructura que dan se muestra en el corte 4 de la figura 5, de dirección NNO-SSE, que los corta casi perpendicularmente. La posición de estos pliegues en la figura 3 no es fácil de dibujar, pues son muy seguidos y quedan muy juntos unos de otros. Sin embargo se puede deducir en parte por la situación del corte 4 en dicha figura; además, la situación de buzamientos invertidos de foliaciones casi E-O, en esta figura, localiza algunos de los pliegues. Su tamaño oscila mucho, con algunos ejes de más de $1 \mathrm{~km}$ de corrida observada, mientras que otros sólo se ven en pequeños afloramientos. Se trata de pliegues apretados, netamente vergentes al NNO, con flancos invertidos que localmente muestran su charnela y el paso a los flancos normales. Son visibles en los dos tramos de mármoles calizos y en el dolomítico se han detectado cuando se pliegan juntamente con los mármoles calizos superiores. La figura 6 corresponde a una composición fotográfica, prácticamente paralela al corte 4 , en la que se han superpuesto los contactos litológicos, con más detalle que en dicho corte, pero hay que tener en cuenta para su comparación las grandes diferencias de escala de la misma, de manera que su sector central se encuentra grandemente aumentado con relación a los sectores más alejados, tanto hacia el fondo como a los lados (esta deformación puede estimarse comparando el ancho de la carretera en los puntos donde se observa). Otros ejemplos de estos pliegues se muestran en las fotos B a C de la figura 4 cuyo aspecto parece indicar una cierta disarmonía en el plegamiento. Son de tamaño decamétrico, de dirección $\mathrm{N} 70^{\circ} \mathrm{E}$ y vergentes al NNO (son generalmente más apretados que los de dirección N-S y se muestran mejor en los cortes).

Se está, por tanto, ante una superposición de pliegues casi ortogonales. En muchos puntos no se podría decir qué juego de pliegues es anterior pero hay otros en los que el orden de superposición es claro. Así, al SO, en las proximidades de la Atalaya, los pliegues casi E-O son plegados por los casi N-S. Esta misma superposición se observa en otros puntos, aunque en la mayoría pase desapercibida y 


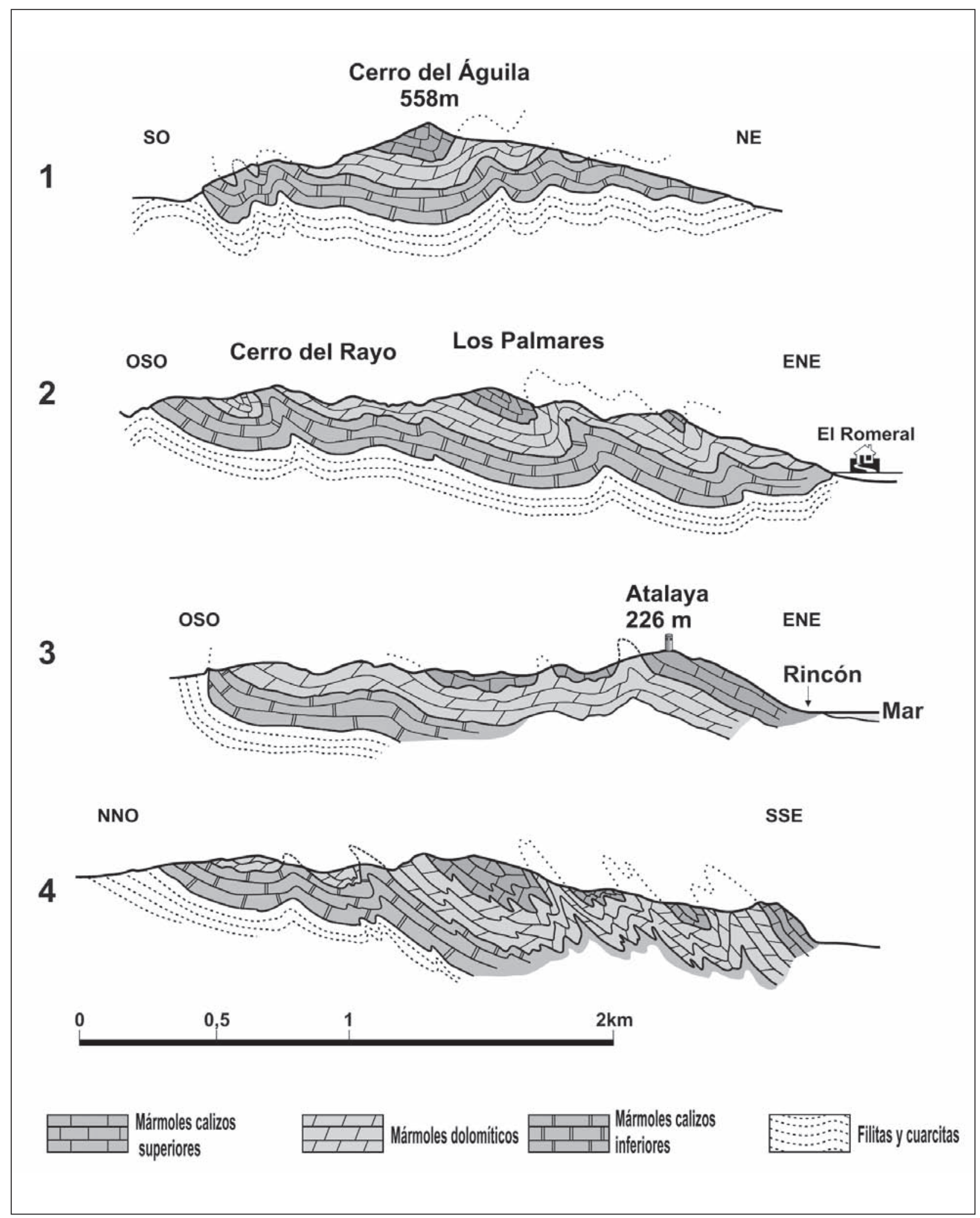

Fig. 5.-Cortes geológicos del sector centro-oriental del área estudiada mostrando la estructura controlada por pliegues casi N-S (cortes 1 a 3) y la de los pliegues casi E-O (corte 4 cuyas partes centro y sur coinciden en buena medida con los rasgos mostrados en la figura 6). 


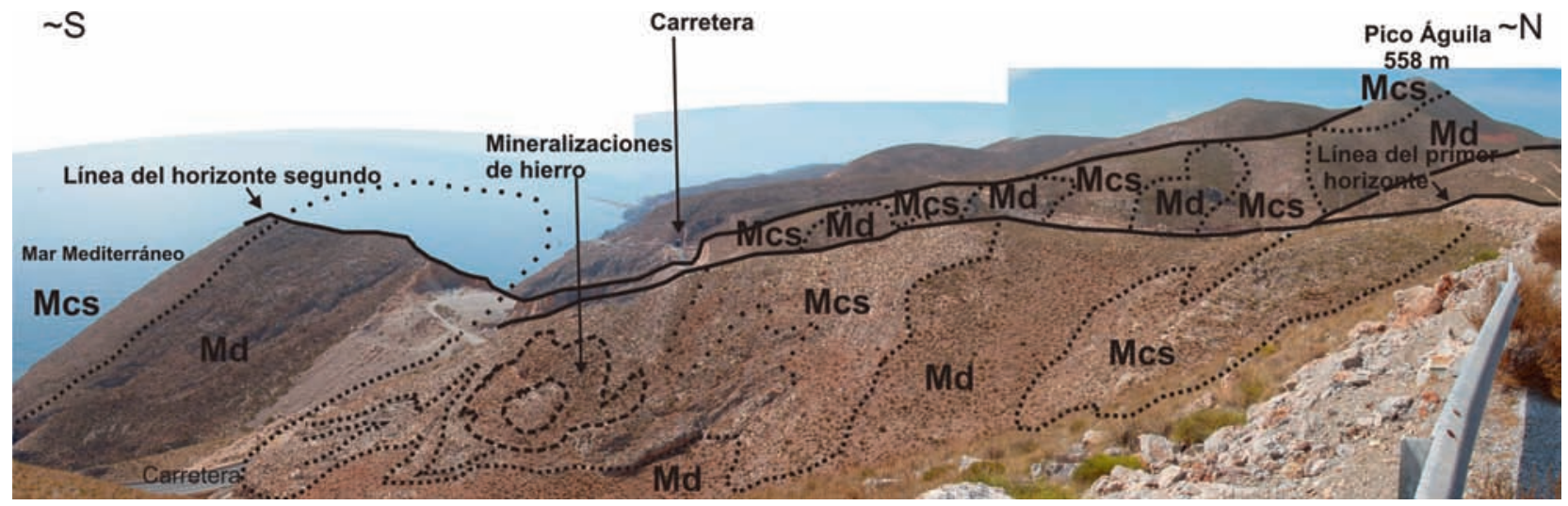

Fig. 6.-Composición fotográfica que muestra el sector situado al oeste de la Atalaya en la que se han introducido los principales contactos geológicos. Md: mármoles dolomíticos, Mcs: mármoles calizos superiores. La escala de la figura varía enormemente según los puntos; es mucho más grande en los próximos y por tanto hay mucha deformación relativa. Se puede tener una idea de esta deformación comparando la anchura de la carretera allí donde se observa.

parece que hay tan sólo pliegues E-O. Pero, si hay un buen corte, los $\mathrm{N}-\mathrm{S}$ se muestran suficientemente bien. Esto ocurre por ejemplo en el talud casi vertical de la ensenada de Calahonda. Por otra parte, algunos pliegues grandes, tal como el del Pico del Águila, tienen direcciones intermedias, de manera que cabe preguntarse si corresponden a una adaptación parcial de anteriores pliegues E-O. Se deduce, por tanto, que el primer plegamiento es el que actualmente tiene una dirección $\mathrm{N} 70^{\circ} \mathrm{E}$ a $\mathrm{E}-\mathrm{O}$ que además da generalmente pliegues más apretados y el segundo es el de dirección aproximada N-S. Es decir, sus ejes son prácticamente perpendiculares. El modelo de interferencia de esta superposición se puede asignar en general al que Ramsay (1977) denomina «tipo 1», pero en algún caso concreto al «tipo 2» con una cierta mayor deformación de los flancos de los pliegues primeros.

\section{Pliegues menores}

Existe una total continuidad en tamaño desde los pliegues que se acaban de describir a los que afectan a pocos niveles, con tamaños inferiores al metro. Están claramente relacionados todos ellos y estos menores presentan también dos direcciones predominantes: una oscila entre $\mathrm{N} 50^{\circ} \mathrm{E}$ y $70^{\circ} \mathrm{E}$, a veces hasta $\mathrm{N} 100^{\circ} \mathrm{E}$ y otra es próxima a la N-S, con direcciones que oscilan entre $\mathrm{N} 150^{\circ}$ a $\mathrm{N} 200^{\circ} \mathrm{E}$ (es decir, $\mathrm{N} 20^{\circ} \mathrm{E}$ ). Sin embargo pueden encontrarse pliegues con cualquier dirección intermedia. En los cortes de la actual carretera de la costa son más abundantes los pliegues de dirección aproximada E-O.
En general es la foliación de los mármoles la que se observa plegada definiendo los pliegues descritos. Normalmente no se observan foliaciones de plano axial salvo en puntos muy apretados de charnelas de pliegues, que en general desaparecen en distancias menores al metro. Localmente se ven superficies de foliaciones con formas alabeadas como adaptándose a las dos direcciones de los pliegues.

También existe concordancia en las vergencias, de manera que los pliegues menores que se aproximan a la dirección E-O tienen neta vergencia al $\mathrm{N}$ y los casi N-S al O. Tan sólo se ha observado una excepción a este hecho y se encuentra próxima al antiguo vertedero que hay al SO de la Atalaya. Allí hay algunos pliegues de dirección E-O que presentan vergencia al S. Se encuentran situados dentro de otros pliegues de mayor tamaño e igual dirección y parecen corresponder a una falta de espacio local que expulsó parte de los materiales hacia el sur.

Estos pliegues menores van acompañados por otras estructuras a veces bien representadas en algunos finos niveles metapelíticos que localmente se intercalan entre los mármoles y que generalmente son tan sólo una simple pátina. Una de estas estructuras corresponde a una linearidad de crenulación recubierta por pequeños cristales de micas blancas. Esta crenulación es paralela a las charnelas de los pliegues, tanto a los E-O como a los casi N-S. En algún punto se observa cómo cambia de dirección y se adapta y en otros se ven las dos direcciones cruzadas. También, en algunos puntos de los mármoles superiores existen unas fibras blancas que podrían corresponder a algún anfíbol del tipo de la tremolita y que pueden observarse solas o con la mica blanca. 

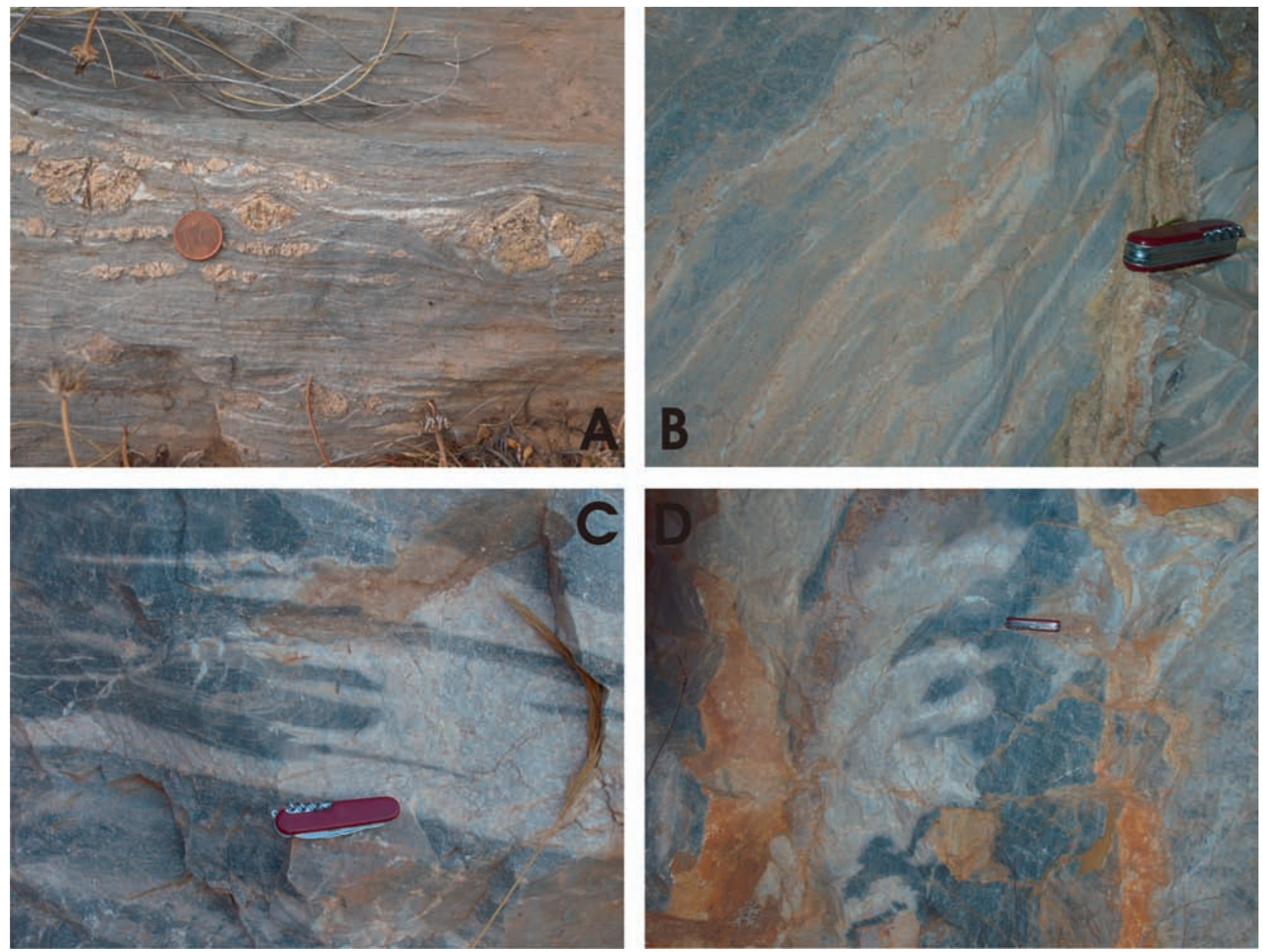

Fig. 7.-A) Ejemplo de budines observados en numerosos pliegues. B a D) Pliegues intrafoliares que muestran un progresivo comportamiento fluidal.

Ésta suele estar orientada mientras que las citadas fibras sólo lo están en parte y en algunos sitios, aunque contenidas en un plano, no tienen ninguna orientación.

Los flancos de los pliegues menores se encuentran en muchos casos estirados de una forma casi fluidal. Existen también budines (fig. 7A) en estos flancos cuya orientación no siempre es la misma, de manera que los cuellos de los budines en algunos casos son paralelos a los ejes de los pliegues pero también hay otros en posición aproximadamente perpendicular. Sin embargo, ambas direcciones, que producirían una estructura a modo de tableta de chocolate, no se han visto en un mismo pliegue, pues generalmente se observan tan sólo cortes parciales de los mismos.

Finalmente hay que indicar que el contacto entre las filitas y los mármoles inferiores no suele presen- tar pliegues menores. Sí lo presentan los mármoles, incluso los calcoesquistos, y las filitas, pero generalmente de forma independiente, sin que se vean implicadas las filitas con los términos más altos. Posiblemente es una cuestión debida a la gran diferencia reológica de los materiales concernidos, lo que implica una cierta disarmonía entre ambas litologías.

\section{Pliegues intrafoliares}

Dentro de la foliación que presentan los mármoles existen también numerosos pliegues (fig. 7B a D). En no pocos casos esos pliegues son coherentes con los pliegues menores que afectan a diversos niveles y con los pliegues mayores, viéndose casos de estiramientos e incluso de budines internos, etc. 
En otros, esa coherencia no parece existir, pues los pliegues aparecen a modo de fragmentos de pliegues anteriores (fig. 7C y D) con una marcada desorganización. En estos casos no se pueden atribuir con seguridad estos fragmentos a ninguno de los dos sistemas de pliegues descritos y cabe la posibilidad de que incluso puedan ser anteriores, pertenecientes a otro sistema anterior, no observado a escala macroscópica. A veces estas deformaciones intrafoliares parecen no existir pero algunos niveles metapelíticos las muestran (fig. 8A).

En los mármoles dolomíticos es difícil observar esas estructuras, tanto de pliegues intrafoliares como budines, estiramientos de flancos, etc., sin que se pueda decir que no existan en absoluto. Aún así, en algunos cortes frescos se observa que carecen de esas estructuras. Incluso, hay algunos niveles con fósiles de algas que no muestran apariencia de deformación interna intensa. Posiblemente esté en relación con la menor plasticidad de este tipo de roca con respecto a los mármoles calizos que existen tanto a techo como a muro de los dolomíticos.

\section{Fracturas}

Prácticamente no hay fracturas que se puedan relacionar con el proceso de plegamiento descrito. Es verdad que es común el desarrollo de fracturas, fallas y diaclasas, singenéticos con la formación de los pliegues tal como señalan en la región Orozco et al. $(1998,2004)$. Sin embargo, entre las muchas fracturas observadas, tan sólo un kilómetro al NNE de Calahonda hay un pequeño cabalgamiento de filitas sobre mármoles que tiene una dirección aproximada N50E. Además de ese rasgo, se debe indicar que el contacto entre las filitas y los mármoles en numerosos puntos se encuentra tectonizado de manera que las filitas han cortado a parte de los mármoles, particularmente en las charnelas de los pliegues anticlinales casi N-S. Esto no se recoge en el mapa de la figura 3.

Hay, sin embargo, un gran número de fracturas afectando a los mármoles, y su tamaño es también muy variable. Algunas fallas son muy pequeñas y afectan tan sólo a un simple nivel de la foliación (fig. 8B). Posiblemente se han formado en el paso de la deformación predominantemente dúctil a la frágil tras la formación de los pliegues casi N-S.

Las fallas de mayor tamaño observadas no exceden del centenar de metros y de acuerdo con sus características se describen según un supuesto orden de formación. a) Hay algunas fallas de direcciones aproximadas $\mathrm{N} 60^{\circ} \mathrm{E}$ con estrías casi horizontales y desplazamientos dextrorsos que denotan la existencia de una compresión aproximadamente E-O. Pero igualmente otras son aproximadamente $\mathrm{N} 150^{\circ}$, dextrorsas, $\mathrm{y}$ $\mathrm{N} 30^{\circ} \mathrm{E}$, sinistrorsas, compatibles con una compresión aproximadamente N-S. Son generalmente métricas y apenas desplazan las estructuras de los pliegues mayores.

b) En algún punto de la carretera de la costa se han observado cuerpos sigmoidales limitados por fallas (fig. 8C, foto hecha aproximadamente a $1 \mathrm{~km}$ al E de Calahonda). La propia geometría de estos cuerpos y las estrías que los acompañan indican que se tratan de cuerpos formados por una extensión posiblemente relacionada con un proceso de levantamiento. Sin embargo, las direcciones de las estrías son variadas lo que puede indicar que cada uno de estos cuerpos se ha movido en diversas direcciones según las condiciones de cada momento.

c) Existen además multitud de pequeñas fallas normales (fig. 8D) de direcciones próximas a la N-S que indican una extensión aproximadamente E-O. Hay otras muchas fallas de diversa orientación y buzamiento.

Es además común la superposición de diversos movimientos en cada plano de falla, superposición que no siempre sigue la misma pauta. Es decir, sobre fallas con movimientos horizontales a veces se ven superpuestos movimientos normales, casi verticales, pero en otros casos sucede exactamente lo contrario.

Otras fracturas, generalmente diaclasas, tienen una disposición casi ubicua en los mármoles. Generalmente se observan como líneas de fractura dibujadas en la superficie de las rocas, pero de ellas casi no se puede sacar más información que la dirección y su buzamiento y a veces ni siquiera el buzamiento de forma fiable. Es en los cortes de las carreteras o en cualquier corte fresco donde se observan mejor. Se han medido numerosas diaclasas, más de 200, que muestran direcciones no siempre coincidentes de unos afloramientos a otros. De ellas, las más abundantes tienen direcciones próximas a la dirección N-S, con oscilaciones del orden de 30 grados tanto hacia el oeste como al este. Generalmente su buzamiento es próximo a la vertical, pero no siempre es así. Son diaclasas de tensión y parecen indicar una extensión aproximadamente E-O. Hay que señalar, sin embargo, que algunos juegos tienen direcciones muy diferentes indicando otras direcciones de extensión. Algunas diaclasas tienen relle- 

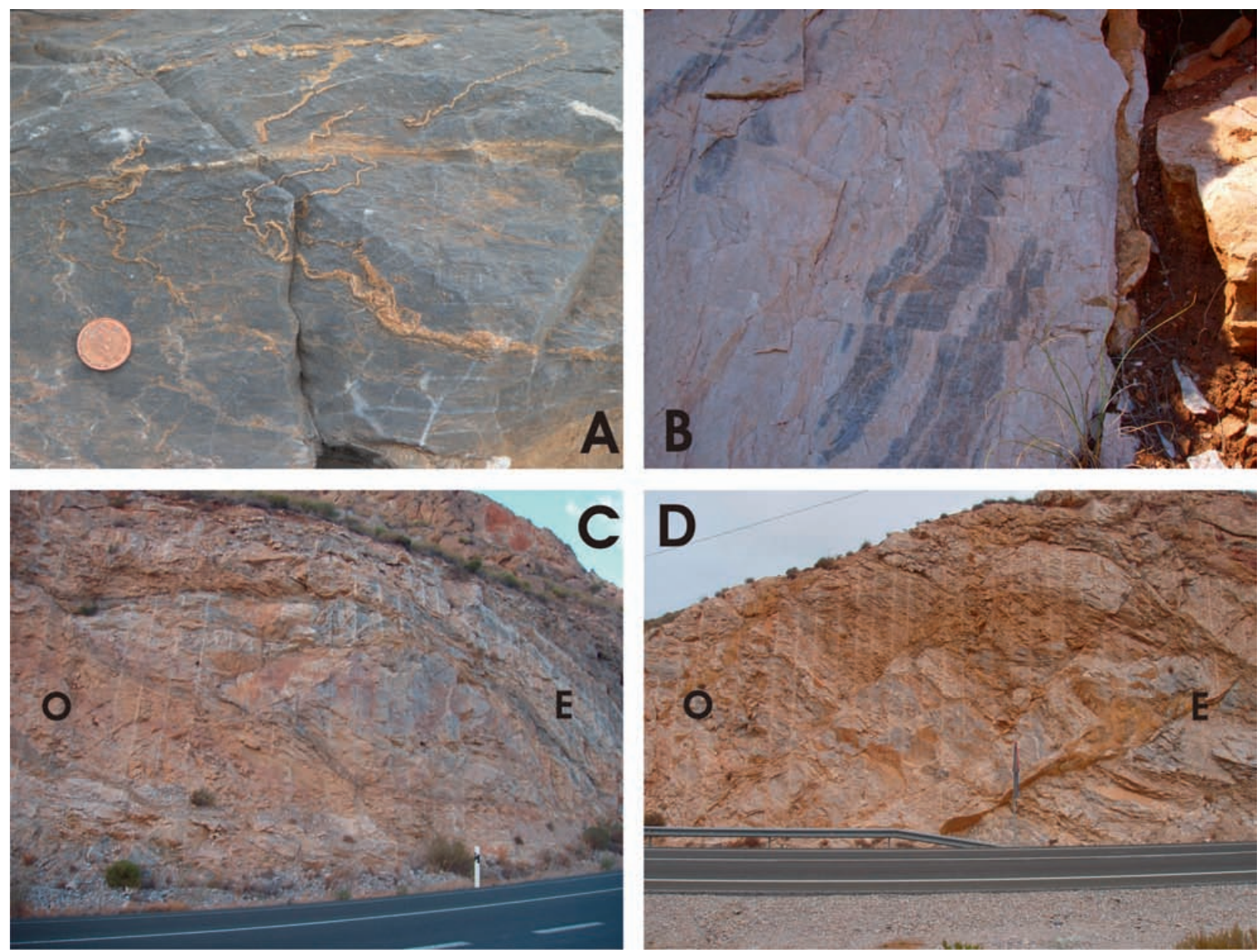

Fig. 8.-A) Niveles metapelíticos que en ocasiones muestran las estructuras existentes en el interior de los niveles de foliación. B) Pequeñas fallas que afectan al interior de los niveles de foliación. C) Cuerpos sigmoidales limitados por fallas de bajo ángulo. D) Fallas normales que indican una extensión ENE-OSO. Las fallas mostradas en C y D en muchos casos presentan estrías que indican diversos movimientos, a veces de diferente carácter.

nos de calcita y parecen corresponder a juegos más antiguos. Los más modernos, con diaclasas más abiertas, no suelen tener rellenos. Su estudio pormenorizado se escapa ahora de los objetivos del presente artículo.

Cuando se observan las diaclasas formadas en el interior de las capas de los mármoles, hay también de dos tipos según se hayan rellenado o no. Generalmente se disponen perpendiculares a la dirección de estiramiento y las rellenas de calcita parecen indicar que se han formado en un momento de mayor plasticidad de la roca.

Finalmente hay que indicar que este macizo marmóreo se está degradando en muchos puntos del contacto con las filitas. Se forman profundas grietas abiertas a veces varios metros con continuidad, en algún caso de centenares de metros. Es un proceso de deslizamiento lento de los mármoles que tienen debajo a las filitas y que van cayendo según la pendiente en bloques que mantienen su cohesión aparente, dado su enorme tamaño. Afecta tanto a los mármoles como a las brechas formadas encima. Su orientación es variable, siempre de acuerdo con la topografía. Estas grietas a veces se sitúan a distancia del contacto, incluso más de $100 \mathrm{~m}$.

\section{Discusión}

Según los datos precedentes, los pliegues de dirección aproximada $\mathrm{N} 70^{\circ} \mathrm{E}$ a E-O son los primeros que se han formado, sin descartar que algunos de los fragmentos de pliegues antes señalados en los pliegues intrafoliares sean previos. Las condiciones en 
que lo hicieron debieron ser las propias de un metamorfismo regional, de manera que los minerales neoformados, fundamentalmente mica blanca que suele aparecer en las superficies de la foliación y a veces en su interior, se adaptan plenamente a las nuevas estructuras. También los granos de calcita de los mármoles recristalizaron y fluyeron.

La segunda etapa corresponde a los pliegues de dirección aproximada N-S. No tenemos datos para saber si esta etapa se produjo de forma casi continua en relación a la anterior o bien si medió un largo lapso de tiempo, aunque estos nuevos pliegues también produjeron deformaciones netamente plásticas en los mármoles.

La existencia de pliegues intrafoliares con trasposición de flancos, budines, etc., indica un neto aplastamiento de la roca según la dirección de compresión y un estiramiento perpendicular en dos direcciones, al menos localmente. Esto es común en diversos mantos del Complejo Alpujárride, de los cuales el estudiado tiene una posición intermedia. Encima quizás estaba ya situado el complejo Maláguide.

Pliegues de dirección $\mathrm{N} 70^{\circ} \mathrm{E}$ a E-O son corrientes en la mayoría de las unidades alpujárrides e incluso en las del Complejo Nevado-Filábride infrayacentes. Parecen estar de alguna forma en relación con el proceso de superposición de los mantos que debió producirse antes del Oligoceno superior (Serrano et al., 2006 y 2007) y su vergencia general es hacia el norte. Por el contrario, los pliegues de dirección próxima a la N-S son mucho menos abundantes, aunque hay ejemplos de ellos.

Los pliegues N-S más cercanos se encuentran directamente al norte, en la parte oriental de la Sierra de Lújar (Estévez et al., 1985) (fig. 2). Esta sierra presenta en ese sector un par de pliegues kilométricos, si bien existe una adaptación progresiva de manera que se pasa a la dirección E-O al S de la sierra. Da la impresión de que hubo una primera formación de pliegues E-O con una compresión casi $\mathrm{N}-\mathrm{S}$ que pasó a ser E-O a ONO-ESE, de manera que en el margen oriental de esa sierra se formaron esos pliegues N-S y a la vez un pliegue curvo que hacía la transición.

En Sierra Tejeda (su posición se indica en la figura 1) existen algunos pliegues kilométricos poco apretados de dirección NO-SE (Sanz de Galdeano, 1989), si bien abundan abrumadoramente los E-O, muy apretados, pero hay numerosos pliegues menores de dirección N-S, además de los E-O y de otros de ejes verticales o casi. Sus característi- cas geométricas son variables pero lo que se destaca en este caso es la existencia de varios sistemas diferentes (Alonso Chaves, 1995). Su superposición da estructuras de interferencia de «tipo 1 », dando formas en «cajas de huevos» a muy distinta escala (Sanz de Galdeano, 1989). De acuerdo con la geometría general observada, los pliegues NOSE son posteriores.

Más al oeste, en las sierras de Mijas y Blanca (posición en fig. 1) igualmente dominan los pliegues de dirección aproximada E-O con vergencia al norte. Sin embargo, los bordes occidentales de ambas sierras presentan pliegues en su conjunto $\mathrm{N}-\mathrm{S}$, si bien vergentes al E. Esto lo interpretan Andreo \& Sanz de Galdeano (1994) y Sanz de Galdeano \& Andreo (1995) como debido a la traslación hacia el oeste de la Zona Interna Bética que se produjo tras su estructuración en mantos, chocando con las unidades de los Flysch y con las zonas externas Bética y Rifeña, que configuraban el antepaís con respecto a la Zona Interna. Por eso, estas sierras tienen en sus bordes occidentales vergencias al E. Sin embargo, la propia Sierra Blanca tiene todo su borde SE invertido con vergencia al $\mathrm{O}$ y por el sur forma un enorme y complejo pliegue invertido curvo. Ocurre un poco al modo del sur de Sierra de Lújar.

Estos rasgos de las sierras Blanca y Mijas se explican bien por la citada traslación hacia el oeste de la Zona Interna Bética que debió iniciarse posiblemente algo antes del Oligoceno superior, en un sector situado bastante cerca de la terminación occidental de la propia Zona Interna Bética. Sin embargo, la existencia de los pliegues casi N-S en un lugar bastante oriental dentro de la cordillera, hace pensar que los efectos de la colisión fueron más extensos de lo que en principio se supuso. Al menos en la transversal Lújar-Calahonda los pliegues N-S allí existentes parecen apoyar esta interpretación. A esto se suma que el sector de Lújar y Calahonda se encuentra justo al este de la línea que va de la terminación occidental de Sierra Nevada (y del complejo Nevado-Filábride) a la costa donde actualmente se localizan fallas recientes (del Mioceno superior a la actualidad) que cortan transversalmente a la Zona Interna (Sanz de Galdeano \& López Garrido, 2000). Quizás el hecho de que las citadas fallas se localicen ahí se deba a la existencia previa de límites o discontinuidades geológicas, tal como el propio borde occidental del Complejo Nevado-Filábride, formando una zona de debilidad relativa, que ya, incluso antes del Mioceno, permitiera una mayor deformación, aun cuando se estu- 
viera lejos del borde occidental del conjunto de la Zona Interna Bética.

Si volvemos a los mármoles de Calahonda-Castell de Ferro, tras la segunda etapa de plegamiento, la presencia del mineral fibroso blanco, quizás tremolita, generalmente no orientado o sólo parcialmente orientado, parece indicar un período en el que la compresión debió disminuir sin que lo hiciera la temperatura. Posiblemente fue el momento en el que se inició la exhumación de la unidad.

Alguna orientación adicional en lo referido a edades de deformación en el Alpujárride se puede tomar de Platt et al. (2005) quienes indican edades de 50 a 30 Ma como edades en las que la recristalización fue intensa en dicho complejo. El valor de 40 a $30 \mathrm{Ma}$ lo interpretan como debido a un enfriamiento gradual. $50 \mathrm{Ma}$ corresponden aproximadamente a los inicios del Eoceno medio y $30 \mathrm{Ma}$ a los inicios del Oligoceno superior. Estas edades encajan bastante bien con las propuestas por otros autores, así De Jong (1991, 1992) y Serrano et al. (2006 y 2007) entre otros y parecen aplicables a las deformaciones de los mármoles ahora estudiados.

Las distintas etapas de fracturación posiblemente guardan el mismo orden expresado al hacer su descripción, de manera que las más antiguas debieron ser aquellas que indican compresiones hacia el oeste, en continuidad de los movimientos de la Zona Interna hacia el oeste y que se pudieron producir durante el Mioceno inferior y medio. A esto se sumó muy pronto un proceso de levantamiento, exhumación de la Zona Interna. El encaje entre los movimientos de fallas hacia el oeste y la exhumación, con el consecuente adelgazamiento cortical, no es claro y en parte pudo ser coetáneo. El caso es que durante la exhumación se produjeron importantes despegues extensionales (GarcíaDueñas et al., 1992) de los que las fallas que dan cuerpos sigmoidales pueden ser testimonio, aunque en el área estudiada no presentan direcciones preferenciales netas.

El resto de fracturas (aunque algunos tipos de diaclasas se asocian a procesos ocurridos en el Mioceno inferior y medio) pueden ser atribuidas al período que va del Mioceno superior a la actualidad. La compresión casi N-S, deducida a partir de algunas fallas conjugadas dextrorsas y sinistrorsas, y la extensión, sobre todo ENE-OSO, que indican otras fallas son compatibles con lo observado en otros sectores de la cordillera (Sanz de Galdeano \& Vera, 1992; Galindo Zaldívar et al., 1993). Igual se puede decir de gran parte de las diaclasas.

\section{Conclusiones}

En los mármoles de Calahonda-Castell de Ferro se distinguen dos juegos de pliegues diferentes. De ellos, los de dirección $\mathrm{N} 70^{\circ} \mathrm{E}$ a E-O son los primeros que se han formado. El segundo juego corresponde a los pliegues de dirección aproximada N-S. Esta secuencia de formación se observa en diversos puntos, particularmente en el sector situado al oeste de la torre Atalaya. No conocemos el tiempo que medió entre la formación de uno y otro juego de pliegues, pero ambos juegos de pliegues se han formado en condiciones en que los mármoles tenían una notoria plasticidad.

Los pliegues $\mathrm{N}^{\circ} 0^{\circ} \mathrm{E}$ a E-O abundan en las unidades de la Zona Interna Bética, lo que no es el caso de los de dirección casi N-S. En las proximidades del área estudiada estos últimos también se observan en la Sierra de Lújar y más al oeste particularmente en Sierra Blanca. Pueden estar relacionados con el desplazamiento de la Zona Interna hacia el oeste y formándose por la resistencia que encontraba esta zona en su movimiento.

En su conjunto, la formación de estos pliegues debió ocurrir desde parte del Eoceno al Oligoceno y quizás la segunda etapa llegue al Oligoceno superior.

La fracturación no afecta a lo esencial de la estructura de estos mármoles de Calahonda. Las fracturas existentes se interpreta que fueron formadas en una secuencia de etapas que van desde la traslación hacia el oeste, una exhumación producida a continuación y una posterior compresión aproximadamente $\mathrm{N}-\mathrm{S}$ junto con una notoria extensión de dirección ENE-OSO. Estas fracturas se han debido producir desde el Mioceno inferior a la actualidad. Las últimas desde el Mioceno superior.

\section{AGRADECIMIENTOS}

A. Caballero delineó las figuras. Este estudio se ha financiado a través de los proyectos CGL2007-60535/BTE, TOPO-IBERIA CONSOLIDER-INGENIO CSD2006-00041, 83/2007 del M.M.A., CGL2008-03249/BTE y P06RNM-01521 de la Junta de Andalucía y el grupo RNM 217 de la Junta de Andalucía. Agradezco las correcciones de la Dra. R. Babín y del Dr. F. Alonso que revisaron el artículo y que, particularmente el segundo, hicieron un minucioso trabajo que sin duda lo ha mejorado.

\section{Referencias}

Aldaya, F. (1969). Los mantos alpujárrides al S de Sierra Nevada (Zona bética, prov. de Granada). Acta Geológica Hispánica, 4: 126-130. 
Aldaya, F. (1981). Mapa Geológico de España, e: 1:50.000, hoja 1056 (Albuñol), Instituto Geológico y Minero de España. 39 pp.

Aldaya, F. \& García Dueñas, V. (1976). Les séquences lithologiques des nappes alpujarrides au Sud et au SudOuest de la Sierra Nevada (Cordillères bétiques, Andalousie). Bulletin de la Societé Géologique de France, 18: 635-639.

Aldaya, F.; García Dueñas, V. \& Navarro Vila, F. (1979). Los Mantos Alpujárrides del tercio central de las Cordilleras Béticas. Ensayo de correlación tectónica de los Alpujárrides. Acta Geològica Hispànica. Homenatge a Lluis Solé i Sabarís, 14: 154-166.

Alonso-Chaves, F.M. (1995). Evolución tectónica de Sierra Tejeda y su relación con procesos de engrosamiento y adelgazamiento corticales en las Cordilleras Béticas. Tesis Univ. Granada, 265 pp.

Andreo, B. \& Sanz de Galdeano, C. (1994). Stratigraphy and structure of the Sierra de Mijas (Alpujarride Complex, Betic Cordillera). Annales Tectonicae. Firenze, 8: 23-35.

Delgado, F.; Estévez, A.; Martín, J.M. \& Martín Algarra, A. (1981). Observaciones sobre la estratigrafía de la formación carbonatada de los mantos alpujárrides (Cordilleras béticas). Estudios Geológicos, 37: 45-57.

De Jong, K. (1991). Tectono-metamorphic studies and radiometric dating in the Betic Cordilleras (SE Spain), with implications for the dynamics of extension and compression in the western Mediterranean area. Tesis doctoral, Amsterdam University, 204 pp.

De Jong, K. (1992). A new Geodynamic Model for the Betic Cordilleras based on P-T-t path and Structural data from the Eastern Betic. Física de la Tierra, 4: 77-107.

Estévez, A.; Delgado, F.; Sanz de Galdeano, C. \& Martín Algarra, A. (1985). Los Alpujárrides al Sur de Sierra Nevada. Una revisión de su estructura. Mediterránea (Alicante), 4: 5-32.

Galindo-Zaldívar, J.; González-Lodeiro, F. \& Jabaloy A. (1993). Stress and palaeostress in the Betic-Rif cordilleras (Miocene to the present). Tectonophysics, 227: 105-126. doi:10.1016/0040-1951(93)90090-7

García-Dueñas, V.; Balanyá, J.C. \& Martínez Martínez, J.M. (1992). Miocene extensional detachments in the outcropping basement of the northern Alboran basin (Betics) and their implications. Geo-Marine Letters, 12: 88-95. doi:10.1007/BF02084917

Orozco, M.; Alonso-Chaves, F.M. \& Nieto, F. (1998). Development of large north-facing folds and their relation to crustal extension in the Alborán domain (Alpujarras region, Betic Cordilleras, Spain). Tectonophysics, 298: 271-295. doi:10.1016/S0040-1951(98)00188-7

Orozco, M.; Álvarez-Valero A.M.; Alonso-Chaves, F.M. \& Platt, J.P. (2004). Internal structure of a collapsed terrain: The Lújar syncline and its significance for the fold- and sheet-structure of the Alborán Domain (Betic
Cordilleras, Spain). Tectonophysics, 385: 85-104. doi:10.1016/j.tecto.2004.04.025

Platt, J.P.; Kelley, S.P.; Carter, A. \& Orozco M. (2005). Timing of tectonic events in the Alpujárride Complex, Betic Cordillera, southern Spain. Journal of the Geological Society, 162: 451-462.

Ramsay, J. (1977). Plegamiento y fracturación de Rocas. H. Blume Ediciones. Madrid. 590 pp.

Sanz de Galdeano, C. (1989). Estructura de las Sierras Tejeda y de Cómpeta (Conjunto Alpujárride, Cordilleras Béticas). Revista de la Sociedad Geológica de España, 2: 77-84.

Sanz de Galdeano, C. (1997). La Zona Interna BéticoRifeña (Antecedentes, unidades tectónicas, correlaciones y bosquejo de reconstrucción paleogeográfica). Monográfica Tierras del Sur. Univ. de Granada, 316 pp.

Sanz de Galdeano, C. \& Andreo, B. (1995). Structure of Sierra Blanca (Alpujarride Complex, West of the Betic Cordillera). Estudios Geológicos, 51: 43-55. doi:10.3989/egeol.95511-2282

Sanz de Galdeano, C. \& López-Garrido, A.C. (1999). Estratigrafía y estructura de las unidades alpujárrides en el borde occidental de Sierra Nevada (Granada, España). Revista de la Sociedad Geológica de España, 12: 187-198.

Sanz de Galdeano, C. \& López-Garrido, A.C. (2000). Las fallas tortonienses a cuaternarias entre Granada y la Costa: el límite occidental del Nevado-Filábride y de las unidades alpujárrides inferiores. Revista de la Sociedad Geológica de España, 13: 519-528.

Sanz de Galdeano, C. \& López-Garrido, A.C. (2003). Revisión de las unidades alpujárrides de las sierras de Tejeda, Almijara y Guájares (sector central de la Zona Interna Bética, provincias de Granada y Málaga). Revista de la Sociedad Geológica de España, 16: 135-149.

Sanz de Galdeano, C. \& Vera, J.A. (1992). Stratigraphic record and palaeogeographical context of the Neogene basins in the Betic Cordillera, Spain. Basin Research, 4: 21-36.

Serrano, F.; Guerra-Merchán, A.; El Kadiri, K.; Sanz de Galdeano, C.; López-Garrido, A.C.; Martín-Martín, M. \& Hlila R. (2007). Tectono-sedimentary setting of the Oligocene-early Miocene deposits on the BeticRifian Internal Zone (Spain and Morocco). Geobios, 40: 191-205. doi:10.1016/j.geobios.2006.04.005

Serrano, F.; Sanz de Galdeano, C.; El kadiri, K.; Guerra Merchán, A.; López-Garrido, A.C.; Martín Martín, M. \& Hlila, R. (2006). Oligocene-early Miocene transgressive cover of the Betic-Rif Internal Zone. Revision of its geological significance. Eclogae Geologicae Helvetiae, 99: 237-253. doi:10.1007/s00015-006-1186-9

Recibido el 8 de junio de 2009 Aceptado el 23 de octubre de 2009 Publicado online el 25 de noviembre de 2009 